\title{
The moderating role of genetics: the effect of length of hospitalization on children's internalizing and externalizing behaviors
}

\author{
Maya Benish-Weisman ${ }^{1 *}$, Eitan Kerem ${ }^{2}$, Ariel Knafo-Noam ${ }^{3}$ and Jay Belsky ${ }^{4}$ \\ ${ }^{1}$ Department of Counseling and Human Development, University of Haifa, Haifa, Israel, ${ }^{2}$ Division of Pediatrics, Hadassah \\ Hospital, Jerusalem, Israel, ${ }^{3}$ Department of Psychology, The Hebrew University of Jerusalem, Jerusalem, Israel, \\ ${ }^{4}$ Department of Human Ecology, University of California Davis, Davis, CA, USA
}

\section{OPEN ACCESS}

Edited by:

Hanna E. Stevens,

University of lowa, USA

Reviewed by:

Mary L. Schneider,

University of Wisconsin-Madison,

USA

lan Kodish,

University of Washington, USA

${ }^{*}$ Correspondence:

Maya Benish-Weisman,

Department of Counseling and Human Development, The University

of Haifa, Mount Carmel,

Haifa 31905, Israel

maya.bw@edu.haifa.ac.il

Specialty section:

This article was submitted to Child and Neurodevelopmental Psychiatry,

a section of the journal

Frontiers in Psychiatry

Received: 20 October 2014

Accepted: 16 July 2015

Published: 17 August 2015

Citation:

Benish-Weisman M, Kerem E, Knafo-Noam A and Belsky J (2015) The moderating role of genetics: the effect of length of hospitalization on

children's internalizing and externalizing behaviors.

Front. Psychiatry 6:109.

doi: 10.3389/fpsyt.2015.00109
The study considered individual differences in children's ability to adjust to hospitalization and found the length of hospitalization to be related to adaptive psychological functioning for some children. Applying the theoretical framework of three competing models of gene-X-environment interactions (diathesis-stress, differential susceptibility, and vantage sensitivity), the study examined the moderating effect of genetics (DRD4) on the relationship between the length of hospitalization and internalizing and externalizing problems. Mothers reported on children's hospitalization background and conduct problems (externalizing) and emotional symptoms (internalizing), using subscales of the 25-item Strength and Difficulties Questionnaire (1). Data on both hospitalization and genetics were available for 65 children, $57 \%$ of whom were females, with an average age of 61.4 months $(S D=2.3)$. The study found length of hospitalization did not predict emotional and behavior problems per se, but the interaction with genetics was significant; the length of hospitalization was related to diminished levels of internalizing and externalizing problems only for children with the 7R allele (the sensitive variant). The vantage sensitivity model best accounted for how the length of hospitalization and genetics related to children's internalizing and externalizing problems.

Keywords: hospitalization, length of hospitalization, emotional problems, behavioral problems, gene-environment interaction, DRD4, externalizing behavior, internalizing behavior

\section{Introduction}

Hospitalization is a challenging experience for young children. It is frequently the sudden and unexpected result of disease or injury, leaving little time for preparation, and is often accompanied by physical discomfort or pain. Evidence reveals enduring effects on children's internalizing and externalizing behaviors post-hospitalization $(2,3)$. Little is known, however, about longer-term effects extending beyond a year. The longer-term legacy of hospitalization is the focus of this report.

The effects of hospitalization, not surprisingly, may vary as a function of amount of time spent in the hospital. As it turns out, however, reported effects of hospitalization length on children's emotional and behavioral functioning are mixed. Whereas some research indicates that longer periods of hospitalization predict more problematic functioning (4-6), other work suggests longer periods of hospitalization which are followed by recovery promote adaptive psychological functioning $(7,8)$. 
Especially notable are the results of a meta-analysis showing that hospitalization exceeding 3 days forecasts less negative behavioral change than do shorter stays of 2-3 days (9). Conceivably, children adjust over time to the new and unfamiliar environment of the hospital. In fact, it has been suggested that hospitalization affords the opportunity for emotional growth (10).

We hypothesize that the contrasting evidence on the effects of hospitalization length in the case of young children could be the result of some children simply being more susceptible to environmental effects than others. Therefore, our research tests the proposition that variation in the effects of hospitalization is likely to be a function not only of length of time spent in the hospital, but also of a child's genetic make-up. The study design follows up a clinical sample at age five, a minimum of 2 years after their discharge, thereby affording us the opportunity to determine whether children with a certain genetic make-up are affected by their hospitalization more than others in terms of internalizing and externalizing behaviors. It is important to note that the correlational design of the study allows conclusions on relations, not causality. Accordingly, it is not clear whether hospitalization influences internalizing/externalizing problems or vice versa. Before providing specific design and measurement details, we turn to conceptual models of gene-X-environment (GXE) interaction and the role of genetics in shaping susceptibility to environmental influences.

\section{Conceptual Models of Gene-X-Environment Interaction}

Most research on GXE interaction - the focus of the current inquiry - has been guided by the diathesis-stress model of environmental action (11). This theoretical perspective stipulates some individuals, due often to their personal characteristics - including their genetic make-up - are more likely to be negatively affected by contextual adversity. Certain genes which predispose individuals to succumb to adversity are regarded as "vulnerability genes" or "risk alleles" (12). Others not carrying these risk alleles are less likely to be negatively affected by contextual adversity and are considered "resilient." In recent years, two alternative models of environmental action which can be applied to GXE have been advanced: differential susceptibility and vantage susceptibility.

\section{Differential Susceptibility}

The differential-susceptibility perspective presumes individual differences in developmental plasticity; that is, some individuals are more susceptible to the environmental regulation of their development than others (13-15). What distinguishes this theoretical model from the diathesis-stress perspective is the presumption that the very individuals whom the latter perspective presumes to be especially susceptible to the negative effects of contextual adversity are also disproportionately likely to benefit from a supportive, enriched, or benign environment. When this way of thinking is applied to the issue of effects of hospitalization, it suggests that for some young children - but not for others - longer periods of hospitalization will have positive effects on emotional and behavioral functioning, whereas shorter periods will have negative effects for some, but not for others. Thus, those carrying "plasticity genes" should, compared to those lacking them, show both more and less problematic functioning depending on whether, respectively, they spend less or more time in the hospital. As previous research suggests, a longer time in the hospital may allow children to adjust as they learn to use the various environmental resources, such as parents and medical staff, to overcome the hospitalization experience (16).

\section{Vantage Sensitivity}

An alternative to diathesis-stress and differential-susceptibility models of GXE interaction has recently been advanced (17). Essentially, it reflects only the "bright side" of differentialsusceptibility thinking and, thus, is the exact opposite of the diathesis-stress model. Instead of conceptualizing some individuals as more susceptible to both positive and negative environmental influences (i.e., differential susceptibility) or to the negative effects of contextual adversity alone (i.e., diathesisstress), "vantage sensitivity" presumes that some individuals are disproportionately likely to benefit from would-be positive environmental effects, while not being especially susceptible to the anticipated negative effects of contextual adversity. In other words, they may be predisposed to benefit from contextual support and enrichment - that is, to be developmentally sensitive to environmental advantage (18). Applied to the issue at hand, it predicts some children, due to their genetic make-up, will benefit more than others from longer hospitalizations, but children with a similar genetic make-up who experience short periods of hospitalization will not be more adversely affected than children carrying different genes.

The ultimate goal of the present inquiry is to determine which of the three theoretical models under consideration best accounts for how a particular gene moderates the effect of hospitalization length on young children's emotional and behavioral functioning.

\section{DRD4}

For many genes, the DNA sequence varies across individuals. These sequences are known as polymorphisms. We focus on a polymorphism in the third exon of the dopamine D4 receptor gene (DRD4-III). This polymorphism has two main variants that differ by the number of 48-base-pair tandem repeats in exon III: 7 present and 7 absence repeat allele. DRD4 is involved in the limbic areas of the brain playing a role in emotional and cognitive functioning. More specifically, variations in this gene across individuals have been related to variations in attentional, motivational, and reward mechanisms (19). Studies have shown that DRD4 interacts with environmental stressors to predict externalizing behaviors among preschoolers concurrently (19, 20) and longitudinally (21). There is also some evidence of the importance of DRD4 to internalizing behaviors $(20,22)$. However, it has been shown that GXE interactions might be limited to specific populations (22) or not exist at all (23), stressing the importance of more studies.

Therefore, we focus on the presence vs. absence of the 7-repeat allele, first because of its relationship to variation in attentional and reward-related mechanisms which could conceivably influence how children respond to long vs. short hospitalizations. Second, we consider this putative "plasticity gene" because it has been found - perhaps more than any other polymorphism 
examined to date - to moderate environmental effects in a manner consistent with differential susceptibility (24).

\section{Materials and Methods}

\section{Participants and Procedure}

Families in this study were drawn from the Longitudinal Israeli Study of Twins (LIST) examining genetic and socialization influences on development. All Jewish families identified by the Israeli Ministry of the Interior as having twins were contacted by mail close to the twins' fifth birthday. Mothers were asked about the twins' hospitalization history and behavior as well as additional information beyond the scope of this report (25). Children were asked to provide a genetic sample with parental permission. Data collection was approved by the Ethics Committees of the Herzog Hospital, Jerusalem, and the Hebrew University. Children who were hospitalized immediately after birth were excluded from the analysis, given their potential for developmental problems (26).

\section{Measures}

Mothers reported on children's conduct problems (externalizing) and emotional symptoms (internalizing) at age five, using subscales of the 25-item Strength and Difficulties Questionnaire (1), with five items comprising each subscale. The mothers rated responses on a scale from $0=($ not true/seldom) to 2 (certainly true/very often). Sample items are as follows: "Often fights with other youth or bullies them" (conduct problems); "Many worries or often seems worried" (emotional symptoms). Cronbach's alpha was 0.62 for conduct problems and 0.64 for emotional symptoms.

\section{Hospitalization History}

Hospitalization history was assessed with questions to the parents about whether the children had been hospitalized, and if so, at what age and for how long. In addition, parents were asked to specify the reason for hospitalization.

\section{DRD4-III Polymorphism}

DNA was extracted by Master Pure kit (Epicentre, Madison WI) and PCR amplification was carried out. The exon III repeat region of the DRD4 receptor was characterized by a PCR amplification procedure (using a Reddy Mix kit, AB gene, Surrey UK) with the following primers: F5'-TTCCTACCCTGCCCGCTCATGC TGCTGCTCATCTGG-3'; R5'-ACCACCACCGGCAGGACC CTCATGGCCTTGCGCTC-3'. We performed PCR reactions using $5 \mu$ l Master Mix (Thermo scientific), $2 \mu$ l primers $(0.5 \mu \mathrm{M})$, $0.6 \mu \mathrm{l} \mathrm{Mg} / \mathrm{Cl} 2(2.5 \mathrm{mM}), 0.4 \mu \mathrm{l}$ DMSO $5 \%$, and $1 \mu \mathrm{l}$ of water to total $9 \mu$ volume; an additional $1 \mu \mathrm{l}$ of genomic DNA was added to the mixture. All PCRs were employed on a Biometra T1 Thermocycler (Biometra, Güttingem, Germany).

\section{Results}

\section{Preliminary Analysis}

Data on both hospitalization and genetics were available for 65 children (65\% with 7R allele, 55\% females; 35\% 7-absent allele,
$60 \%$ females), average age of 61.4 months $(\mathrm{SD}=2.3)$. The extent of hospitalization ranged from 1 to 10 days $(M=4.08, \mathrm{SD}=2.67)$; $51.4 \%$ of the children were hospitalized between the ages of 1 and 12 months and $48.6 \%$ between the ages of 13 and 36 months. $28.8 \%$ were hospitalized for infections, $19.7 \%$ for surgical operations, $13.6 \%$ for diarrhea, and $9.1 \%$ for other problems; $28.8 \% \mathrm{did}$ not answer this question. Children hospitalized more than once $(n=5)$ were excluded from the analyses.

The DRD4-III 7-repeat allele was not associated with the length of hospitalization ( $r=0.07$, n.s.). This rules out the possibility that this genetic characteristic might be directly related to the length of hospitalization and that any detected GXE interaction could be an artifact of gene-environment correlation involving this polymorphism. We found no relationship between the cause of hospitalization and the length of hospitalization $[\mathrm{F}(4)=2.41$, n.s.], enabling us to claim that the following results are hospitalizationlength related and not hospitalization-reason related.

In addition, we found no main effect of sex or interaction of sex with DRD4 for conduct problems ( $B=-0.1$, n.s., $B=-38$, n.s., respectively) or for emotional symptoms $(B=0.22$, n.s., $B=-0.36$, n.s., respectively).

Similarly, there was no main effect of the age of hospitalization or the interaction of the age of hospitalization with DRD4 for conduct problems ( $B=-0.06$, n.s., $B=0.03$, n.s., respectively) or for emotional symptoms $(B=-0.14$, n.s., $B=-0.47$, n.s., respectively).

\section{Effect of Length of Hospitalization and Genetics on Problems}

We examined the pathways between length of hospitalization and internalizing and externalizing behaviors with DRD4 as a moderator in children aged five using Mplus (Muthén \& Muthén, 1998-2007). Two models were tested, one for emotional (internalizing) problems and one for conduct (externalizing) problems.

\section{Internalizing Behaviors}

The model for emotional symptoms fit the data well (comparative fit index $[\mathrm{CFI}]=1$, root mean square error of approximation $[$ RMSEA $]=0)$. The length of hospitalization was not associated with emotional symptoms $(\beta=-0.14, \mathrm{SE}=0.14$, Est./S.E. $=-1.02$, n.s.), although children with $7 \mathrm{R}$ allele experienced more emotional symptoms than those without it $(\beta=3.15, \mathrm{SE}=0.87$, Est./S.E. $=3.63, p=0.00)$. Importantly, the interaction between DRD4-III and length of hospitalization was significant $(\beta=-2.88$, $\mathrm{SE}=0.92$, Est. $/$ S.E. $=-3.14, p=0.002$ ). Thus, the slope reflecting the relationship between length of hospitalization and emotional symptoms was calculated for each genetic sub-group and tested for significance (27). Whereas length of hospitalization proved unrelated to emotional symptoms in the case of children not carrying the $7 \mathrm{R}$ allele ( $\beta=-0.024$, n.s.), for those carrying it, longer hospitalization predicted fewer emotional symptoms $(\beta=1.27$, $p=0.01$ ) (see Figure 1)

\section{Externalizing Behaviors}

The model to predict conduct problems at age five fits the data well $(\mathrm{CFI}=0.94$, RMSEA $=0.06)$. As we found for emotional 


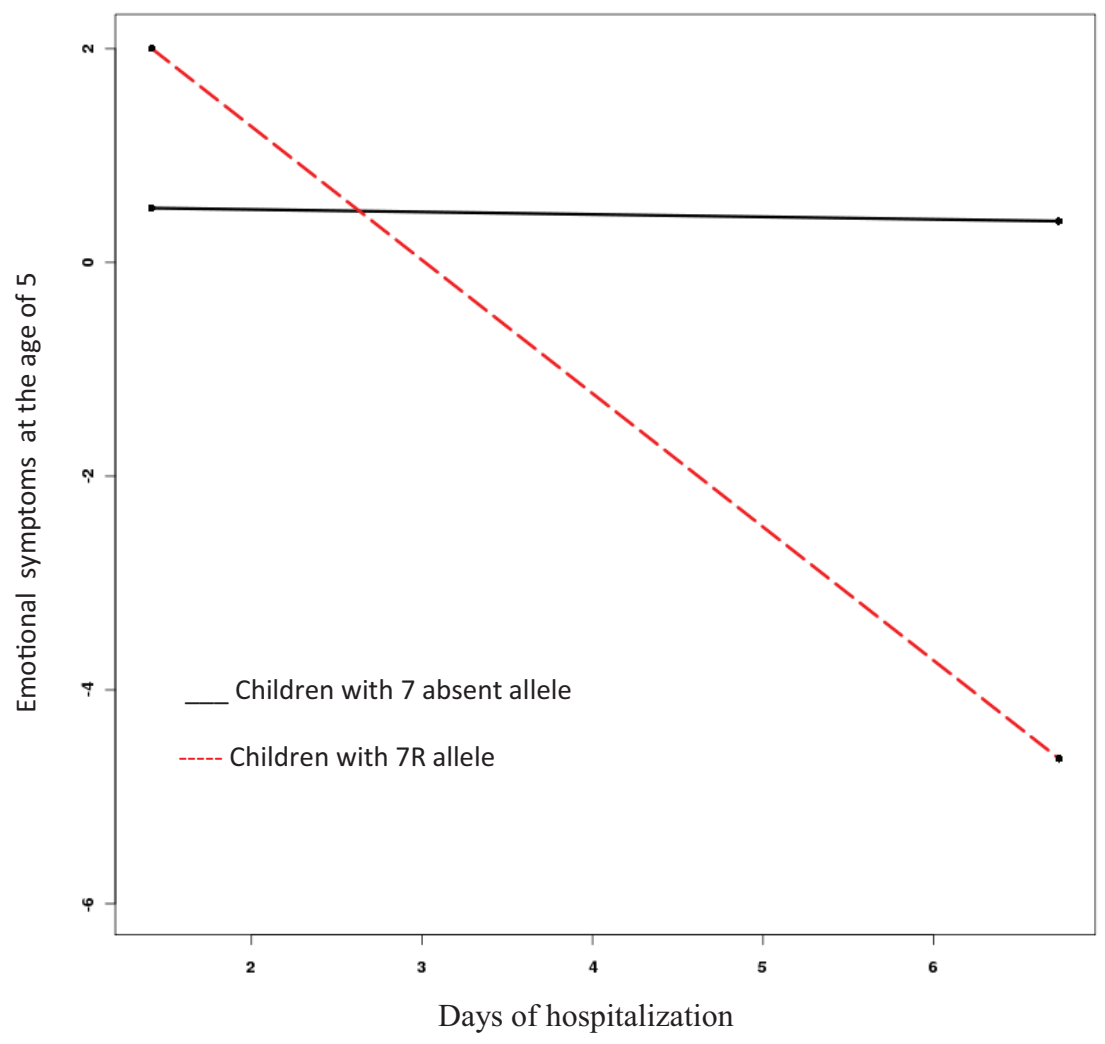

FIGURE 1 | Interaction effect of length of hospitalization and presence or absence of DRD4-III 7-repeat allele on emotional symptoms at age five.

symptoms, length of hospitalization did not predict conduct problems at the age of five $(\beta=-0.13, \mathrm{SE}=0.11$, Est./S.E. $=-1.14$, n.s.). Nevertheless, children with 7-present allele had more conduct problems than those with 7 -absent allele $(\beta=4.12, \mathrm{SE}=0.59$, Est./S.E. $=6.96, p=0.00)$. Furthermore, the interaction between DRD4-III and length of hospitalization was significant in predicting conduct problems among 5 -year-olds $(\beta=-4.19, \mathrm{SE}=0.59$, Est./S.E. $=-7.16, p=0.00$ ) (see Figure 2); specifically, for the children carrying the $7 \mathrm{R}$-allele, more time in hospital predicted fewer conduct problems.

\section{Discounting Alternative Possibilities and Choosing Best Fitting Conceptual Model}

The analyses above are typical of studies of GXE interaction. Recently, however, there have been calls to conduct more rigorous tests to distinguish among conceptual models of GXE interaction (28-30).

This approach (29) addresses certain limitations in GXE interaction examination approaches. The first is the visual inspection limitation. Some previous studies have used different ranges of the independent variable (X, hospitalization length in the current study) to probe the interaction; others have not specified the range. If there are no standard criteria, different graphs can be sketched from the same data, leading to dissimilar or even contradictory conclusions. We followed the recommendation (29) to set extended interactions boundaries within the range of \pm 1.5 SDs on X; this captured $93 \%$ of the sample and set a rigid criterion, greatly increasing the possibility that the results would be attributed to the appropriate GXE model.

The second limitation addressed in this approach (29) concerns non-linearity. Specifically, in cases where there is a non-linear predictor-outcome relationship, a diathesis-stress effect can be interpreted incorrectly as consistent with the differential susceptibility model. To rule out the possibility of non-linear relation between predictors and outcome, we tested X2 (the independent variable squared) and ZX2 (the moderator doubled the squared independent variable) in predicting emotional symptoms and conduct problems. (We refer to regression model $\mathrm{Y}=\mathrm{b} 0+\mathrm{b} 1 \mathrm{X}+\mathrm{b} 2 \mathrm{Z}+\mathrm{b} 3 \mathrm{XZ}$ where $\mathrm{X}$ represents days of hospitalization and $\mathrm{Z}$ is the dichotomous moderator, DRD4.) We found that the relationship is indeed linear. We found no significant effect for emotional symptoms $(\beta=-0.02, \mathrm{SE}=0.16$, Est./S.E. $=-1.4$, n.s.; $\beta=.-0.27, \mathrm{SE}=0.26$, Est./S.E $=-1.04$, n.s. $)$ or for conduct problems $(\beta=-0.06, \mathrm{SE}=0.15$, Est./S.E. $=-0.39$, n.s.; $\beta=.-0.16, \mathrm{SE}=0.29$, Est./S.E $=-0.55$, n.s.).

The third limitation (29) is the lack of standard index for the quantification of GXE models. To test which conceptual model fit our data, we calculated the Proportion of Interaction (PoI) index (29); this reflects the proportion of the total area of an interaction plot bounded by, in our case, \pm 1.5 SDs on $\mathrm{X}$ that is uniquely attributable to the "better" side of the interaction. PoI values close to 1.0 support the vantage sensitivity model; PoI values close to 0.50 support the differential susceptibility model; and values closer to 0.00 support the diathesis-stress model. One of the 


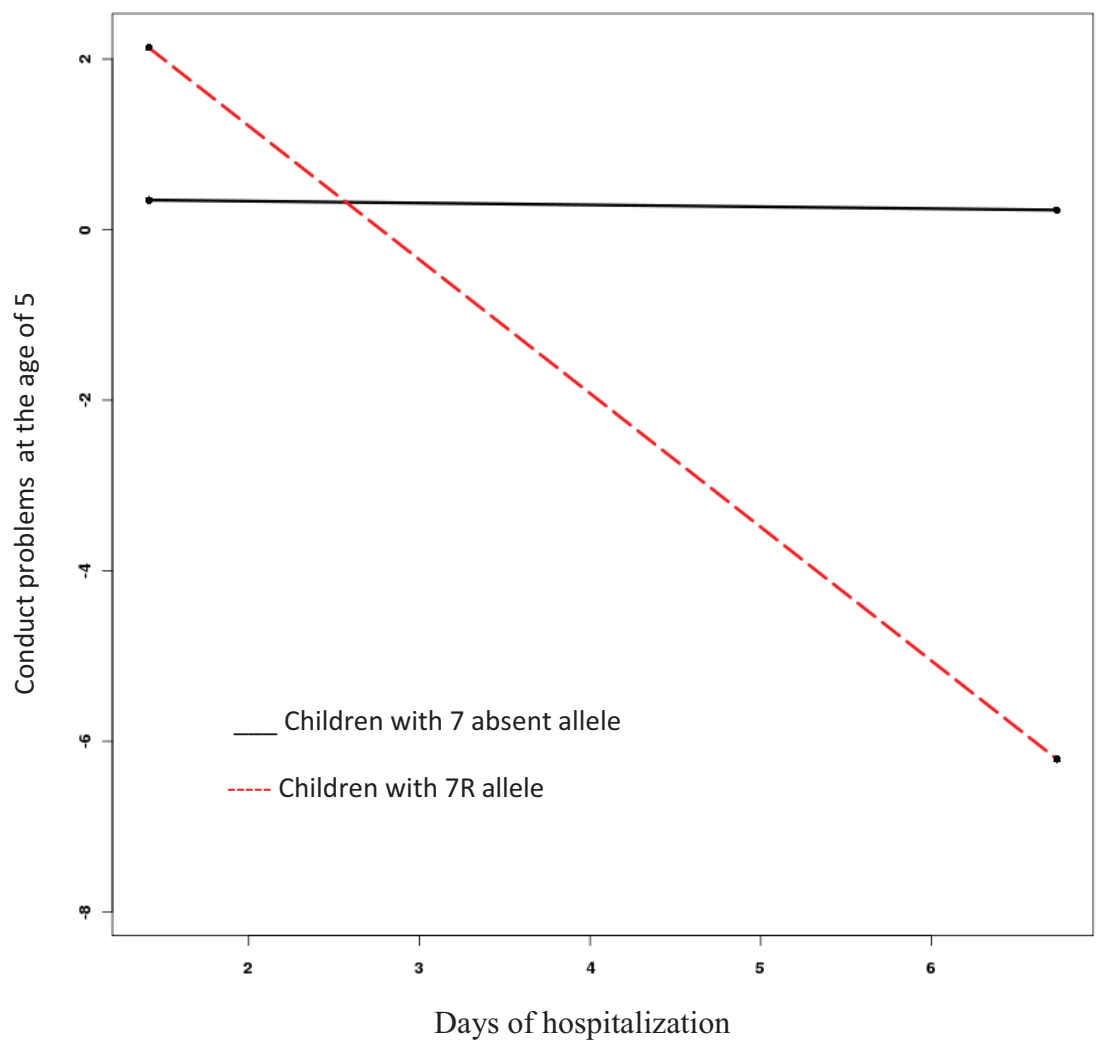

FIGURE 2 | Interaction effect of length of hospitalization and presence or absence of DRD4-III 7-repeat allele on conduct problems at age five.

main advantages of this index is that PoI is not directly affected by sample size as can be seen by the formula below. $\left(\mathrm{B}_{3} \mathrm{X}_{\text {low }}+\mathrm{B}_{2}\right)$ and $\left(\mathrm{B}_{3} \mathrm{X}_{\text {high }}+\mathrm{B}_{2}\right)$ are the simple slopes of $\mathrm{Y}$ (the dependent variable) on $\mathrm{Z}$ the moderator) at low and high $\mathrm{X}$, respectively.

$$
\mathrm{Pol}=\left(\frac{B_{3} \mathrm{X}_{\mathrm{low}}+b_{2}}{B_{3} \mathrm{X}_{\mathrm{high}}+b_{2}}\right)^{-1}
$$

In our study, the PoI values were 0.98 and 0.82 for emotional symptoms and conduct problems, respectively. These results support the vantage sensitivity model over the differentialsusceptibility or diathesis-stress models.

\section{Discussion}

Hospitalization may have a major impact on children's lives, including long-term effects on their development $(2,3)$. Although many previous studies have focused on short-term emotional and behavioral problems, our study followed oncehospitalized children for a much longer term. While all children were studied at age five, their period of hospitalization ranged from 2 to 4 years previously. Findings indicate that length of hospitalization in and of itself does not predict behavioral and emotional problems. Apparent effects - in this observational/ correlational study - of length of hospitalization emerged only when a particular feature of children's genetic make-up was taken into account.
Longer periods of hospitalization predicted fewer internalizing and externalizing behaviors at age five only for those children carrying the $7 \mathrm{R}$ allele. In fact, rather than detecting evidence consistent with either the diathesis-stress (11) or differential-susceptibility models (19-21) of GXE interaction, the results proved consistent with vantage sensitivity (29). That is, we did not find that the length of hospitalization was related to negative outcomes (diathesis-stress model) or that shorter periods of hospitalization were related to negative outcomes and longer ones to positive outcomes (differential sensitivity). This result challenges former findings that see hospitalization as only adverse $(2,3)$. Of course, most previous studies measured children's reactions close in time to the hospitalization experience, while in our study, children's behavior was measured 2 to 4 years after the hospitalization. It is possible that after a certain period of time, the overall experience of hospitalization was remembered as positive rather than negative for children carrying the $7 \mathrm{R}$ allele as we explain below.

The DRD4-III 7-repeat allele has repeatedly been shown to be associated with stronger relations between environmental variables and developmental outcomes, with this effect more pronounced in positive environments (17). We found the length of hospitalization was related to diminished levels of internalizing and externalizing problems among genetically sensitive children. A possible interpretation of the findings is that longer periods of hospitalization benefit sensitive children, at least in terms of their emotional-behavioral well-being. Arguably, longer hospitalization offers these children more opportunity to overcome 
the initial distress; they may gain comfort and a sense of control, resulting in a greater sense of security and, thus, develop fewer problems as the hospital routine becomes familiar and they adjust to a supportive environment that includes caring medical professionals and other hospital staff. They may also come to better understand the nature of their hospitalization experience. Graphs 1 and 2 reveal that the critical time for $7 \mathrm{R}$ allele children to adjust to hospitalization is the second or the third day. These results confirm former meta-analysis (9) showing hospitalization exceeding 3 days forecasts less negative behavioral change than do shorter stays of 2-3 days.

Parenting practices may need to be considered to gain further insight into why these particular findings emerged $(31,32)$. During hospitalization, young children often enjoy close parental care all day long and at night as well. Conceivably, then, longer periods of hospitalization may provide children with extra parental support and care, thereby promoting their emotional security and well-being. Future research should not only take into account variation in children's susceptibility to environmental influences, but also the reasons for hospitalization and the nature of parental care during time in the hospital.

The findings are in line with some studies examining the effect of hospitalization on children's emotional and behavioral problems $(7,8)$ but not with others $(4-6)$. These mixed results may be accounted for by cultural differences in the prevalence of specific alleles across cultures or sub-cultures; that is, the sensitive allele might be frequent in some cultures but not in others. We suggest that in some cultures more children will be sensitive to environmental influence, resulting in different effects on children's externalizing and internalizing problems post hospitalization (33). Most previous studies lack specification of participants' cultural background; therefore, future studies should include this information. Another possible explanation is that the same genetic variation will have a different phenotype in

\section{References}

1. Goodman R. The Strengths and Difficulties Questionnaire: a research note. J Child Psychol Psychiatry (1997) 38(5):581-6. doi:10.1111/j.1469-7610.1997. tb01545.x

2. Rennick JE, Johnston CC, Dougherty G, Platt R, Ritchie JA. Children's psychological responses after critical illness and exposure to invasive technology. J Dev Behav Pediatr (2002) 23(3):133-44. doi:10.1097/00004703-200206000-00002

3. Small L, Melnyk BM. Early predictors of post-hospital adjustment problems in critically ill young children. Res Nurs Health (2006) 29(6):622-35. doi:10.1002/ nur.20169

4. Connolly D, McClowry S, Hayman L, Mahony L, Artman M. Posttraumatic stress disorder in children after cardiac surgery. J Pediatr (2004) 144(4):480-4. doi:10.1016/j.jpeds.2003.12.048

5. Fahrenfort JJ, Jacobs EA, Miedema S, Schweizer AT. Signs of emotional disturbance three years after early hospitalization. J Pediatr Psychol (1996) 21(3):353-66. doi:10.1093/jpepsy/21.3.353

6. Yap JN-K. The effects of hospitalization and surgery on children: a critical review. J Appl Dev Psychol (1988) 9(3):349-58. doi:10.1016/0193-3973(88)90035-4

7. Hefferon K, Grealy M, Mutrie N. Post-traumatic growth and life threatening physical illness: a systematic review of the qualitative literature. $\mathrm{Br} J$ Health Psychol (2009) 14(2):343-78. doi:10.1348/135910708X332936

8. Benish-Weisman M, Wu LM, Weinberger-Litman SL, Redd WH, DuHamel $\mathrm{KN}$, Rini C. Healing stories: narrative characteristics in cancer survivorship narratives and psychological health among hematopoietic stem cell different cultures (23). Future studies should expand the results to include more cultures, allowing us to determine whether they are universal or culture specific.

Despite the evident strengths of the current work, including the focus on genetic moderation of long-term "effects" of hospitalization and the effort to distinguish, via formal statistical criteria, the GXE model that best fits the data, this inquiry has limits. To begin with, the sample is not large, so future work would be well advised to use larger samples while seeking to replicate the findings reported here. An additional limitation is the exclusive focus on child behavior problems; it would be ideal if future work could examine a positive, not just a negative function (and/or its absence). Might the hospitalization experience have fostered the development of self-esteem, for example? Finally, the current design does not allow us to conclude causality. We have shown that length of hospitalization and genetic vulnerability may be related to the symptoms of the child at a later age, but causality should be examined in future studies that will test children before and after hospitalization.

As we note in this article, and as many others have similarly noted, hospitalization can be a major event for children. Whereas previous studies have highlighted the behavioral difficulties that follow hospitalization, this investigation sheds light on the brighter side of hospitalization by applying a candidate-gene and GXE approach to the inquiry.

\section{Acknowledgments}

The authors are indebted to the parents and twins in the Longitudinal Israeli Study of Twins (LIST) for making the study possible. We thank the research assistants who collected and coded the data. LIST is supported by grant No. 31/06 from the Israel Science Foundation and by Starting Grant no. 240994 from the European Research Council (ERC) to AK-N.

transplant survivors. Palliat Support Care (2014) 12(4):261-7. doi:10.1017/ S1478951513000205

9. Thompson RH, Vernon DTA. Research on children's behavior after hospitalization: a review and synthesis. J Dev Behav Pediatr (1993) 14(1):28-35. doi:10.1097/00004703-199302000-00005

10. Shields MG. E-Business and ERP: Rapid Implementation and Project Planning. New York, NY: Wiley (2004).

11. Caspi A, Sugden K, Moffitt TE, Taylor A, Craig IW, Harrington H, et al. Influence of life stress on depression: moderation by a polymorphism in the 5-HTT gene. Science (2003) 301(5631):386-9. doi:10.1126/science.1083968

12. Rutter M. Genes and Behavior: Nature-Nurture Interplay Ex-Plained. London: Blackwell (2006).

13. Belsky J, Pluess M. Beyond diathesis stress: differential susceptibility to environmental influences. Psychol Bull (2009) 135(6):885. doi:10.1037/ a 0017376

14. Ellis BJ, Boyce WT, Belsky J, Bakermans-Kranenburg MJ, Van IJzendoorn MH. Differential susceptibility to the environment: an evolutionary-neurodevelopmental theory. Dev Psychopathol (2011) 23(1):7. doi:10.1017/ S0954579410000611

15. Kim-Cohen J, Gold AL. Measured gene-environment interactions and mechanisms promoting resilient development. Curr Dir Psychol Sci (2009) 18(3):138-42. doi:10.1111/j.1467-8721.2009.01624.x

16. Horowitz L, Kassam-Adams N, Bergstein J. Mental health aspects of emergency medical services for children: summary of a consensus conference. J Pediatr Psychol (2001) 26(8):491-502. doi:10.1093/jpepsy/26.8.491 
17. Pluess M, Belsky J. Vantage sensitivity: individual differences in response to positive experiences. Psychol Bull (2012) 139(4):901-16. doi:10.1037/ a0030196

18. Manuck SB. Delay discounting covaries with childhood socioeconomic status as a function of genetic variation in the dopamine D4 receptor (DRD4). Paper presented at the Society for Research in Child Development, Montreal, QC (2011).

19. Bakermans-Kranenburg MJ, Van IJzendoorn MH, Pijlman FT, Mesman J, Juffer F. Experimental evidence for differential susceptibility: dopamine D4 receptor polymorphism (DRD4 VNTR) moderates intervention effects on toddlers' externalizing behavior in a randomized controlled trial. Dev Psychol (2008) 44(1):293-300. doi:10.1037/0012-1649.44.1.293

20. Schmidt LA, Fox NA, Hamer DH. Evidence for a gene-gene interaction in predicting children's behavior problems: association of serotonin transporter short and dopamine receptor D4 long genotypes with internalizing and externalizing behaviors in typically developing 7-year-olds. Dev Psychopathol (2007) 19(04):1105-16. doi:10.1017/S0954579407000569

21. Windhorst DA, Mileva-Seitz VR, Linting M, Hofman A, Jaddoe VW, Verhulst FC, et al. Differential susceptibility in a developmental perspective: DRD4 and maternal sensitivity predicting externalizing behavior. Dev Psychobiol (2015) 57(1):35-49. doi:10.1002/dev.21257

22. Propper C, Willoughby M, Halpern C, Carbone M, Cox M. Parenting quality, DRD4, and the prediction of externalizing and internalizing behaviors in early childhood. Dev Psychobiol (2007) 49(6):619-32. doi:10.1002/dev.20249

23. Sonuga-Barke EJ, Oades RD, Psychogiou L, Chen W, Franke B, Buitelaar J, et al. Dopamine and serotonin transporter genotypes moderate sensitivity to maternal expressed emotion: the case of conduct and emotional problems in attention deficit/hyperactivity disorder. J Child Psychol Psychiatry (2009) 50(9):1052-63. doi:10.1111/j.1469-7610.2009.02095.x

24. Bakermans-Kranenburg MJ, Van IJzendoorn MH. Gene-environment interaction of the dopamine D4 receptor (DRD4) and observed maternal insensitivity predicting externalizing behavior in preschoolers. Dev Psychobiol (2006) 48(5):406-9. doi:10.1002/dev.20152

25. Knafo A. The Longitudinal Israeli Study of Twins (LIST): children's social development as influenced by genetics, abilities, and socialization. Twin Res Hum Genet (2006) 9(6):791-8. doi:10.1375/twin.9.6.791
26. Cole FS, Alleyne C, Barks JDE, Boyle RJ, Carroll JL, Dokken D, et al. NIH Consensus Development Conference statement: inhaled nitric-oxide therapy for premature infants. Pediatrics (2011) 127(2):363-9. doi:10.1542/ peds.2010-3507

27. Aiken LS, West SG. Multiple Regression: Testing and Interpreting Interactions. Newbury Park, CA: Sage (1991).

28. Belsky J, Pluess M, Widaman KF. Confirmatory and competitive evaluation of alternative gene-environment interaction hypotheses. J Child Psychol Psychiatry (2013) 54(10):1135-43. doi:10.1111/jcpp.12075

29. Roisman GI, Newman DA, Fraley RC, Haltigan JD, Groh AM, Haydon KC. Distinguishing differential susceptibility from diathesis-stress: recommendations for evaluating interaction effects. Dev Psychopathol (2012) 24(02):389-409. doi:10.1017/S0954579412000065

30. Widaman KF, Helm JL, Castro-Schilo L, Pluess M, Stallings MC, Belsky J. Distinguishing ordinal and disordinal interactions. Psychol Methods (2012) 17(4):615-22. doi:10.1037/a0030003

31. Coleman WL, Garfield C. Fathers and pediatricians: enhancing men's roles in the care and development of their children. Pediatrics (2004) 113(5):1406-11. doi:10.1542/peds.113.5.1406

32. Higham S, Davies R. Protecting, providing, and participating: fathers' roles during their child's unplanned hospital stay, an ethnographic study. J Adv Nurs (2013) 69(6):1390-9. doi:10.1111/j.1365-2648.2012.06131.x

33. Way BM, Lieberman MD. Is there a genetic contribution to cultural differences? Collectivism, individualism and genetic markers of social sensitivity. Soc Cogn Affect Neurosci (2010) 5(2-3):203-11. doi:10.1093/scan/nsq059

Conflict of Interest Statement: The authors declare that the research was conducted in the absence of any commercial or financial relationships that could be construed as a potential conflict of interest.

Copyright (c) 2015 Benish-Weisman, Kerem, Knafo-Noam and Belsky. This is an open-access article distributed under the terms of the Creative Commons Attribution License (CC BY). The use, distribution or reproduction in other forums is permitted, provided the original author(s) or licensor are credited and that the original publication in this journal is cited, in accordance with accepted academic practice. No use, distribution or reproduction is permitted which does not comply with these terms. 